\title{
Zeitschriftenverzeichnis nach Namen
}

Åarboger for nordisk oldkyndighed og = historie

Abhandlungen der Geistes- und Sozialwissenschaftlichen Klasse.

Akademie der Wissenschaften und der Literatur in Mainz

Bayerische Akademie der Wissenschaften. = Philosophische-historische Klasse.

Abhandlungen

Abhandlungen der Deutschen Akademie = der Wissenschaften zu Berlin

Abhandlungen der Sächsischen Akademie = der Wissenschaften zu Leipzig. Philologisch-historische Klasse

Abr-Nahrain. An annual published by the = Department of Middle Eastern Studies, University of Melbourne

Accademie e biblioteche d'Italia

Acme. Annali della Facoltá di lettere e = filosofia dell'Universitá degli studi di Milano

Acta Academiae Aboensis

Acta ad archaeologiam et artium historiam pertinentia

Acta ad archaeologiam et artium historiam pertinentia. Series altera in $8^{\circ}$

Acta antiqua Academiae scientiarum = hungaricae

Acta archaeologica Academiae scientiarum hungaricae

Acta archaeologica carpathica

Acta archaeologica. Kobenhavn

Acta archaeologica Lovaniensia

Acta Associationis internationalis Terra Antiqua Balcanica

Acta classica. Proceedings of the Classical = Association of South Africa

Acta classica Universitatis scientiarum Debreceniensis

Acta historica. Societas academica dacoromana

Acta hyperborea. Danish studies in classical archaeology

Acta Instituti romani Finlandiae

Acta Musei Napocensis

Acta Musei Porolissensis
AarbKøb

AbhMainz

AbhMünchen

AbhBerlin

AbhLeipzig

Abr-Nahrain

AcBibl

Acme

ActaAcAbo

ActaAArtHist

ActaAArtHist s. a

ActaAntHung

ActaArchHung

ActaACarp

ActaArch

ActaALov

TerraAntBalc

ActaCl

ActaClDebrec

ActaHistDac

ActaHyp

$=$

$=$

$=\quad$ ActaMusNapoca

$=\quad$ ActaMusPorol
Acta numismàtica (Barcelona)

Acta orientalia Academiae scientiarum hungaricae

Acta philologica. Societas academica dacoromana

Acta praehistorica et archaeologica

Acta Universitatis Nicolai Copernici. Archaeologia

Acta Universitatis Nicolai Copernici. = Historia (Torun)

Advances in Archaeological Method and = Theory

Aegaeum. Annales d'archéologie égéenne $=$ de l'Université de Liège

Aegyptiaca helvetica

Aegyptus

Aevum antiquum

Aevum. Rassegna di scienze storiche linguistiche e filologiche

Africa. Institut national d'archéologie et = d'art, Tunis

Ägypten und Levante. Zeitschrift für ägyptische Archäologie und deren Nachbargebiete

Alba Regia. Annales Musei Stephani Regis

Alma mater studiorum

Almanach der Wiener Akademie der Wissenschaften

'Alon mahleqat ha-'atiqot šel medinat Jisra'el

Alt-Thüringen

Altamura. Bollettino dell'ArchivioBiblioteca-Museo civico

Das Altertum

Alto medioevo

Altorientalische Forschungen

American Journal of Ancient History

American Journal of Archaeology

American Journal of Numismatics

American Journal of Philology

American Studies in Papyrology

Ampurias

Anadolu (Anatolia). Ankara
ActaNum

ActaOrHung

ActaPhilSocDac

ActaPraehistA

ActaTorunA

Acta'TorunHist

AMethTh

Aegaeum

AegHelv

Aegyptus

AevumAnt

Aevum

Africa

ÄgLev

Alba Regia

AlmaMaterSt

AlmanachWien

AlonJisrael

AltThür

Altamura

Altertum

AltoMed

Altor $F$

AmJAncHist

AJA

AmJNum

$\mathrm{AJPh}$

AmStP

Ampurias

Anadolu 
Anadolu araştırmaları. Jahrbuch für

kleinasiatische Forschung

Anadolu medeniyetleri müzesi yıllığı =

Anagennesis. A papyrological journal =

Anais do município de Faro

Analecta Bollandiana

Analecta romana Instituti danici

Anales de filología clásica. Universidad de = Buenos Aires

Anales de prehistoria y arqueología. Universidad de Murcia

Anales del Instituto de estudios Gerundenses

Anali Zavoda za povijesne znanosti Istraživačkog centra Jugoslavenske akademije znanosti i umjetnosti $u$ Dubrovniku

Anas. Museo nacional de arte romano de = Mérida

Anatolian Studies

Anatolica

The Ancient History Bulletin

Ancient India

Ancient Society

The Ancient World

Annales archéologiques arabes syriennes

Les annales des Pays Nivernais

Annales d'Ethiopie

Annales du Service des antiquités de = l'Egypte

Annales. Economies, sociétés, civilisations

Annales Universitatis scientiarum Budapestinensis de Rolando Eötvös nominatae. Sectio classica

Annali Benacensi

Annali del Seminario di studi del mondo classico, Sezione di archeologia e storia antica. Napoli

Annali della Facoltà di lettere e filosofia = della Università di Cagliari

Annali della Facoltà di lettere e filosofia. = Università Bari

Annali della Facoltà di lettere e filosofia. = Università di Macerata

Annali della Facoltà di lettere e filosofia, = Università degli studi di Perugia

Annali della Facoltà di lettere e filosofia, = Università di Napoli

Annali della Facoltà di magistero dell'Università di Cagliari

Annali della Fondazione per il Museo = "Claudio Faina"

Annali della Scuola normale superiore di = Pisa

Annali dell'Università di Lecce. Facoltà di $=$ lettere e filosofia e di magistero

Annali. Istituto italiano di numismatica =

Annali. Istituto orientale di Napoli=

L'année épigraphique =

Annuaire de l'Institut de philologie et = d'histoire orientales et slaves
AnadoluAras

AnadoluYil

Anagennesis

AnMunFaro

AnalBolland

AnalRom

AnFilCl

AnMurcia

AnGerona

AnDubr

Anas

AnatSt

Anatolica

AncHistB

AncInd

AncSoc

AncWorld

AAS

AnnNivern

AnnEth

ASAE

AnnEconSocCiv

AnnUnBud

AnnBenac

AnnAStorAnt

AnnCagl

AnnBari

AnnMacerata

AnnPerugia

AnnNap

AnnCaglMag

AnnFaina

AnnPisa

AnnLecce

AnnIstItNum

AnnOrNap

$\mathrm{AE}$

AIPhOr
Annual Byzantine Studies Conference. Abstracts of Papers

Annual Egyptological Bibliography =

The Annual of the American Schools of = Oriental Research

The Annual of the British School at = Athens

Annual of the Department of Antiquities = of Jordan

The Annual of Leeds University Oriental = Society

Annual Report of the Director of the Department of Antiquities, Republic of Cyprus

The Annual Report of the Fogg Art Museum

The British School of Archaeology at = Athens. Annual report of the managing comittee

Annual Report. The Society for Libyan = Studies

Annual (Report). Worcester Art Museum =

Annuario. Accademia etrusca di Cortona =

Annuario bibliografico di archeologia =

Annuario della Accademia delle scienze di = Torino

Annuario della Accademia nazionale dei = Lincei

Annuario della Pontificia accademia = romana di archeologia

Annuario della Scuola archeologica di = Atene e delle Missioni italiane in

Oriente

Annuario. Istituto giapponese di cultura = in Roma

Annuario. Liceo-ginnasio statale G. = Palmieri, Lecce

Antaeus. Mitteilungen des Archäologischen Instituts der Ungarischen Akademie der Wissenschaften

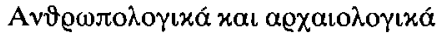
xอovixá

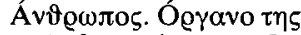

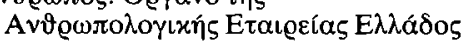

Antichità Pisane

Antichthon. Journal of the Australian= Society for Classical Studies

Antike Kunst

Antike und Abendland

Antike Welt. Zeitschrift für Archäologie = und Kulturgeschichte

Antipolis. A Journal of Mediterranean = Archaeology

Antiqua. Pubblicazione bimestrale= dell'Archeoclub d'Italia per la conoscenza, la tutela e la valorizzazione dei beni culturali

The Antiquaries Journal

L'antiquité classique

Antiquités africaines

Antiquités nationales. Saint-Germain-en- = Laye

Antiquity

Antiquity and Survival

Anuario de historia del derecho español =

Anuario de historia económica y social=

Anzeiger für die Altertumswissenschaft =
AnnByzConf

AnnEgBibl

AASOR

BSA

AAJ

AnnLeedsUnOrSoc

AnnRepCypr

AnnRepFoggArtMus

AnnRepBrSAth

RepSocLibSt

AnnWorcArtMus

AnnAcEtr

AnnBiblaModena

AnnAcTorino

AnnuarioAcLinc

AnnPontAcRom

ASAtene

AnnIstGiapp

AnnuarioLecce

Antaeus

AnthrAChron

Anthropos

AntPisa

Antichthon

AntK

AuA

AW

Antipolis

Antiqua

Ant J

AntCl

AntAfr

AntNat

Antiquity

AntSurv

AnHistDer

AnEcSoc

AnzAW 
Anzeiger. Österreichische Akademie der = Wissenschaften, Wien

Apollo. Bollettino dei musei provinciali = del Salernitano

Apollo. The international magazine of the $=$ arts

Apulum. Acta Musei Apulensis =

Aquileia chiama. Bollettino trimestrale= della Associazione nazionale per Aquileia

Aquileia nostra

Aquitania. Une revue inter-régionale d'archéologie

Arabian Archaeology and Epigraphy

Arbeits- und Forschungsberichte zur sächsischen Bodendenkmalpflege

Archaeologia austriaca

Archaeologia Cantiana

Archaeologia geographica

Archaeologia jugoslavica

Archaeologia Mosellana

Archaeologia or Miscellaneous Tracts Relating to Antiquity Published by the Society of Antiquaries of London

Archaeologia polona

Archaeologiai értesitó

The Archaeological Advertiser

Archaeological and Historical Studies. The Archaeological Society of Alexandria.

Archaeological News

The Archaeological News Letter

Archaeological Reports

Archaeology. A magazine dealing with the antiquity of the world

Archaeometry. Bulletin of the Research = Laboratory for Archaeology and History of Art. Oxford University

Archaeonautica

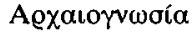

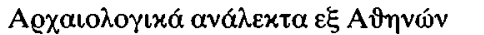

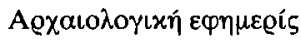

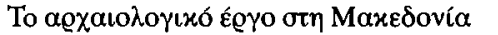

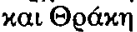

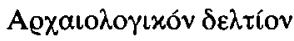

Archäographie. Archäologie und elektronische Datenverarbeitung

Archäologie des Kantons Solothurn

Archäologie der Schweiz.

Mitteilungsblatt der Schweizerischen

Gesellschaft für Ur- und Frühgeschichte

Archäologie und Naturwissenschaften =

Archäologische Ausgrabungen in Baden- = Württemberg

Archäologische Informationen.

Mitteilungen zur Ur- und

Frügeschichte

Das archäologische Jahr in Bayern

Archäologische Nachrichten aus Baden =

Archäologischer Anzeiger =

Archäologisches Korrespondenzblatt =

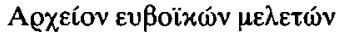

AnzWien

Apollo

ApolloLond

Apulum

Aquileia chiama

AquilNost

Aquitania

ArabAEpigr

ArbFBerSächs

AAustr

ACant

AGeo

AJug

AMosel

Archaeologia

APol

AErt

AAdv

AHistStAlex

ANews

ANL

ARepLond

Archaeology

Archaeometry

Archaeonautica

Archaiognosia

AAA

AEphem

AErgoMak

ADelt

Archäographie

ASoloth

ASchw

ANaturwiss

AAusgrBadWürt

AInf

AJahrBay

AMI

ANachrBad

AA

AKorrBl

ArchEubMel

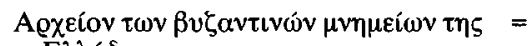

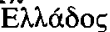

Archeo. Attualità del passato

$=$

Archeografo triestino

Archeologia e calcolatori

Archeologia classica

Archeologia corsa

Archeologia in Campania. Bolletino di informazioni a cura della

Soprintendenza archeologica delle province di Napoli e Caserta

Archeologia medievale. Cultura materiale, insediamenti, territorio

Archeologia moldovei

Archeologia. Rivista bimestrale. Roma

Archeologia. Rocznik Instytutu historii kultury materialnej Polskiej akademii nauk

Archeologia e società

Archeologia. Trésor des âges

Archeologia veneta

Archeologia viva

Archeologičeskij sbornik.

Gosudarstvennyj ordena Lenina

Ermitaž

Archeologické rozhledy

Archeologični pamjarki URSR

Archéologie du Nil Moyen

Archéologie en Bretagne. Bulletin d'information

Archeologija. Akademija nauk Ukrains'koi RSR, Institut archeologii

Archeologija. Organ na Archeologičeskija = institut i muzej pri Bălgarskata akademija na naukite

Architectura. Zeitschrift für Geschichte der Baukunst

Archiv für Begriffsgeschichte

Archiv für Orientforschung

Archiv für Papyrusforschung und verwandte Gebiete

Archív orientální

Archivio della Società romana di storia patria

Archivio storico per la Calabria e la Lucania

Archivio storico per la Sicilia orientale

Archivio storico pugliese

Archivo de prehistoria levantina

Archivo español de arqueología

Arctos. Acta philologica fennica

Arethusa

Argo. Narodni muzej v Ljubljani

Arheološki pregled. Arheološko društvo Jugoslavije

Arheološki radovi i rasprave

Arheološki vestnik (Ljubljana)

Arion

Arqueología. Memoria de las actuaciones = programadas en el año $19 x x$

$O$ arqueólogo português
ArchByzMnem

Archeo

ArcheogrTriest

ACalc

ArchCl

ACors

ACamp

AMediev

AMold

ArcheologiaRoma

ArcheologiaWarsz

ASoc

ArcheologiaParis

AVen

AViva

ASbor

ARozhl

APamKiiv

ANilMoy

ABret

ArcheologijaKiiv

ArcheologijaSof

Architectura

ArchBegriffsGesch

AfO

ArchPF

ArOr

ArchStorRom

ArchStorCal

ArchStorSicOr

ArchStorPugl

ArchPrehistLev

ArchEspA

Arctos

Arethusa

Argo

APregl

ARadRaspr

AVes

Arion

Arqueología

APort 
Arquivo de Beja. Boletim, estudos, arquivo

Ars georgica

Arsberättelse

Arse. Boletín del Centro arqueológico Saguntino

The Art Bulletin

Art et archéologie en Rhône-Alpes

Art Journal

Arte antica e moderna

Arte lombarda

Artibus Asiae

Arts in Virginia

Assaph. Studies in Art History

Assyriological Miscellanies

Atene e Roma

Athenaeum. Studi periodici di letteratura $=$ e storia dell'antichità. Università di Pavia

'Atiqot. English series

Atlal. The journal of Saudi Arabian= archaeology

Atti. Centro di ricerche storiche, Rovigno =

Atti. Centro ricerche e documentazione = sull'antichità classica

Atti de la Accademia delle scienze dell'Istituto di Bologna. Memorie

Atti dei Civici musei di storia ed arte di Trieste. Atti del Museo civico di antichità

Atti della Accademia delle scienze dell'Istituto di Bologna. Rendiconti

Atti della Accademia delle scienze di Torino

Atti della Accademia di scienze, lettere e = arti di Palermo

Atti della Accademia Pontaniana

Atti della Accademia Roveretana degli Agiati. Contributi della classe di scienze umane, di lettere ed arti.

Atti della Società per la preistoria e protostoria della regione Friuli Venezia Giulia

Atti dell'Accademia nazionale dei Lincei. = Classe di scienze morali, storiche e filologiche. Rendiconti

Atti. Istituto veneto di scienze, lettere ed $=$ arti

Atti e memorie della Società dalmata di = storia patria

Atti e memorie della Società istriana di archeologia e storia patria Atti e memorie della Società Magna
Grecia

Atti e memorie della Società tiburtina di = storia e d'arte

Atti e memorie dell'Academia toscana di = scienze e lettere "La Colombaria"

Atti e memorie. Deputazione di storia = patria per le antiche provincie modenesi

Atti e memorie. Deputazione di storia = AttiMemBologna patria per le province di Romagna
ArqBeja

ArsGeorg

Arsberättelse

Arse

ArtB

ArtARhône

ArtJ

ArtAntMod

ArtLomb

ArtAs

Assaph

AssyrMisc

AeR

Athenaeum

'Atiqot

Atlal

AttiRovigno

AttiCAntCl

MemBologna

AttiMusTrieste

RendBologna

AttiAcTorino

AttiPalermo

AttiAcPontan

AttiAcRov

AttiSocFriuli

RendLinc

AttiVenezia

AttiMemDal

AttiMemIstria

AttiMemMagnaGr

AttiMemTivoli

AttiMemFirenze

AttiMemModena
ArtVirg
Aula orientalis. Revista de estudios del Próximo Oriente antiguo

Ausa (Vic)

Ausgrabungen und Funde in WestfalenLippe

Ausgrabungen und Funde.

Nachrichtenblatt für Vor- und

Frühgeschichte

Ayasofia müzesi yıllı̆̆ı. Annual of Ayasofya Museum

Badische Fundberichte

Baetica. Estudios de arte, geografía e historia

Baghdader Mitteilungen

Bajo Aragón. Prehistoria

Balcanoslavica

Balkan Studies

Bayerische Akademie der Wissenschaften. = Philosophisch-historische Klasse.

Sitzungsberichte

Bayerische Vorgeschichtsblätter

Bedi Kartlisa. Revue de kartvélologie

Beiträge zur allgemeinen und vergleichenden Archäologie

Beiträge zur Namenforschung

Beiträge zur Sudanforschung

Bellas artes (Madrid)

Belleten. Türk tarih kurumu

Benàcus. Museo archeologico della Val Tenesi

Beni culturali e ambientali. Sicilia

Berceo (Logroño)

Bericht der Bayerischen

Bodendenkmalpflege

Bericht der Deutschen

Forschungsgemeinschaft

Bericht der Römisch-germanischen Kommission

Berichte über die Verhandlungen der Sächsischen Akademie der Wissenschaften zu Leipzig

Berichten van de Rijksdienst voor het oudheidkundig bodemonderzoek

Berliner Beiträge zur Archäometrie

Berliner Blätter für Vor- und Frühgeschichte

Berliner Jahrbuch für Vor- und Frühgeschichte

Berliner Museen

Berliner numismatische Zeitschrift

Berytus. Archaeological Studies

The Biblical Archaeologist

Bibliotheca classica orientalis

Bibliotheca orientalis

Biblos-Press

Biogradski zbornik

Blätter für Münzfreunde und Münzforschung

Boletín arqueológico. Real sociedad arqueológica tarragonese

Boletín Auriense
AulaOr

Ausa

AusgrFuWestf

AusgrFu

AyasofyaMüzYıl

BadFuBer

Baetica

$\mathrm{BaM}$

BajoAr

Balcanoslavica

BalkSt

SBMünchen

BayVgBl

BediKart

BeitrAllgA

BeitrNamF

BeitrSudanF

BelArt

Belleten

Benàcus

BCASic

Berceo

BerBayDenkmPfl

BerDF

BerRGK

BerVerhLeipz

BerOudhBod

BerlBeitrArchäom

BerlBIVFrühGesch

BerlJbVFrühGesch

BerlMus

BerlNumZ

Berytus

Bibla

BiblClOr

$\mathrm{BiOr}$

Biblos-Press

BiogrZbor

BlMüFreundeF

BATarr

BAur 
Boletín de la Asociación española de amigos de la arqueología

Boletín de la Asociación española de = orientalistas

Boletín de bellas artes. Sevilla

Boletín de la Comision provincial de monumentos históricos y artísticos de Lugo

Boletín de la Institución Fernán Gonzáles, Burgos

Boletín de la Institución „Sancho el = Sabio"

Boletín de la Real academia de Córdoba = de ciencias, bellas letras y nobles artes

Boletín de la Real academia de la historia =

Boletín del Instituto de estudios= giennenses

Boletín del Istituto de estudios helénicos. Universidad de Barcelona, Facoltad de filosofía y letras

Boletín del Museo arqueológico nacional =

Boletín del Museo de Cádiz

Boletín del Seminario de estudios de arte $\mathbf{y}=$ arqueología, Universidad de Valladolid

Bollettino annuale. Musei ferraresi

Bollettino. Centro di studi filologici e = linguistici siciliani

Bollettino d'arte

Bollettino dei classici

$=$

Bollettino dei Musei comunali di Roma =

Bollettino del Centro di studi per la storia = dell'architettura

Bollettino del Circolo numismatico = napoletano

Bollettino del Museo civico di Padova =

Bollettino della Società piemontese di= archeologia e belle arti

Bollettino della Unione storia ed arte $=$

Bollettino dell'Istituto centrale del = restauro

Bollettino dell'Istituto di filologia greca, = Università di Padova

Bollettino dell'Istituto di storia e di arte = del Lazio meridionale

Bollettino di archeologia =

Bollettino di informazioni. Associazione = archeologica "Centumcellae"

Bollettino di numismatica

Bollettino di studi latini

Bollettino. Monumenti, musei e gallerie = pontificie

Bollettino storico della Basilicat

Bollettino storico della città di Foligno =

Bonner Jahrbücher des Rheinischen Landesmuseums in Bonn und des Vereins von Altertumsfreunden im Rheinlande

Boreas. Münstersche Beiträge zur Archäologie

Bracara Augusta. Revista cultural da Camara municipal de Braga

Bremer archäologische Blätter
BEspA

BEspOr

BBelArt

BLugo

BBurgos

BVitoria

BCord

BAcHist

BJaén

BInstEstHel

BMusMadr

BMusCadiz

BVallad

BAnnMusFerr

BFilLingSic

BdA

$\mathrm{BCl}$

BMusRom

BArchit

BCircNumNap

BMusPadova

BTorino

BStorArt

BRest

BFilGrPadova

BLazioMerid

BA

BInfCentumcellae

BNumRoma

BStLat

BMonMusPont

BBasil

BFoligno

$\mathrm{BJb}$

Boreas

BracAug

BremABl
Brigantium. Museo arqueolóxico e histórico

Britannia. A journal of Romano-British = and kindred studies

The British Museum Quarterly

The British Museum Yearbook

Budapest régiségei

Bulletin (annuel) de l'Ecole antique de Nimes

Bulletin archéologique du Comité des travaux historiques et scientifiques. Antiquités nationales

Bulletin archéologique de Provence

Bulletin archéologique du Comité des travaux historiques et scientifiques

Bulletin. Cercle d'études numismatiques =

Bulletin d'archéologie algérienne

Bulletin d'archéologie marocaine

Bulletin d'archéologie sud-est européenne $=$

Bulletin de correspondance hellénique=

Bulletin de l'Association Guillaume Budé =

Bulletin de l'Association Pro Aventico=

Bulletin de liaison. Centre d'études des = peintures murales romaines

Bulletin de l'Institut français

d'archéologie orientale

Bulletin de l'Institut historique belge de = Rome

Bulletin de la Société archéologique champenoise

Bulletin de la Société d'archéologie copte $=$

Bulletin de liaison de la Société des amis de la Bibliothèque Salomon Reinach

Bulletin de la Société française de numismatique

Bulletin de la Société nationale des antiquaires de France

Bulletin des antiquités luxembourgeoises $=$

Bulletin des Musées royaux d'art et d'histoire, Bruxelles

Bulletin des travaux de l'Institut national = d'archéologie et d'art (Tunis). Comptes rendus

Bulletin d'études orientales

Bulletin d'études préhistoriques alpines

Bulletin d'information de l'Association internationale pour l'étude de la mosaïque antique

Bulletin du Centre de recherches et d'enseignement de l'antiquité, Angers

Bulletin du Centre international d'étude des textiles anciens

Bulletin du laboratoire du Musée du Louvre

Bulletin du Musée d'anthropologie préhistorique de Monaco

Bulletin du Musée de Beyrouth arts

Bulletin du Musée national de Varsovie =

Bulletin. The Hebrew University, = Jerusalem, Rabinowitz Fund
Brigantium

Britannia

BMQ

BrMusYearbook

BudReg

BEcAntNimes

BAAntNat

BAProv

BAParis

BCercleNum

BAAlger

BAMaroc

BASudEstEur

$\mathrm{BCH}$

BAssBudé

BProAvent

BPeintRom

BIFAO

BBelgRom

BSocAChamp

BACopt

BSocBiblReinach

BNumParis

BAntFr

BAntLux

BMusBrux

BTravTun

BEtOr

BPréhistAlp

BAssMosAnt

BAngers

BTextilAnc

BLaborMusLouvre

BMusMonaco

BMusBeyrouth

BMusHongr

BMusVars

BJerus 

University of London

Bulletin. J. Paul Getty Museum of Art = (Malibu)

Bulletin monumental

Bulletin. Museum of Fine Arts, Boston

Bulletin of the American Schools of Oriental Research

The Bulletin of the American Society of = Papyrologists

Bulletin of the Ancient Orient Museum = (Tokyo)

Bulletin of the Anglo-Israel Archaeological Society

Bulletin of the Asia Institute

The Bulletin of the Cleveland Museum of $=$ Art

Bulletin of the Egyptological Seminar (New York)

Bulletin of the Institute of Archaeology, = University of London

Bulletin of the Rhode Island School of Design. Museum notes

Bulletin of the Royal Ontario Museum of = Archaeology. University of Toronto

Bulletin. Smith College Museum of Art

Bulletin. Société archéologique d'Alexandrie

Bulletin van de Vereeniging tot bevordering der kennis van de antieke beschaving te 's-Gravenhage

Bulletin Zavoda za likovne umjetnosti= Jugoslavenske akademije znanosti i umjetnosti

Bullettino del Centro Camuno di studi= preistorici

Bullettino del Museo della civiltà romana =

Bullettino della Commissione archeologica comunale di Roma

Bullettino dell'Istituto di diritto romano = "Vittorio Scialoja“

Bullettino di paletnologia italiana

Bullettino senese di storia patria prehistòria $i$ arqueologia de la Diputació provincial de Barcelona

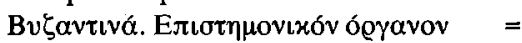

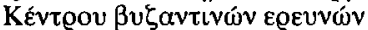

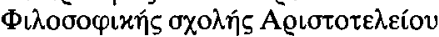

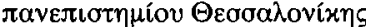

Byzantinisch-neugriechische Jahrbücher =

Byzantinische Forschungen.

Internationale Zeitschrift für Byzantinistik

Byzantinische Zeitschrift

Byzantino-bulgarica

Byzantinoslavica

Byzantion

Caesaraugusta. Publicaciones del Seminario de arqueología y

numismática aragonesas

Caesarodunum
Bulletin. Institute of Classical Studies,

Butlletí informatiu de l'Institut de

BICS

BulletinMalibu

BMon

BMusFA

BASOR

BAmSocP

BAncOrMus

BAngloIsrASoc

BAsInst

BClevMus

BEgNewYork

BALond

BProvidence

BOntMus

= BulletinNorthampton

$=\quad$ BArchAlex

$=\quad$ BABesch

$=$

BLikUm

BCamuno

BMusCivRom

BCom

BDirRom

BPI

BSiena

BABarcel

Byzantina

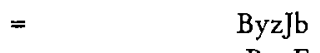

ByzF

ByzZ

$=$

$=$ Byzantino-Bulgarica

$=\quad$ Byzantinoslavica

$=\quad$ Byzantion

XXX

Caesarodunum
Cahiers archéologiques

Cahiers d'archéologie subaquatique

Cahiers de Byrsa

Cahiers de la céramique égyptienne

Cahiers de la Délégation française en Iran

Cahiers de Karnak

Les cahiers de Mariemont

Cahiers de recherches de l'Institut de papyrologie et d'égyptologie de Lille.

Sociétés urbaines en Egypte et au Soudan

Cahiers de Tunisie

Cahiers du Groupe de recherches sur

l'armée romaine et les provinces

Cahiers du Musée Champollion. Histoire $=$ CahMusChampollion et archéologie

Cahiers ligures de préhistoire et d'archéologie

Cahiers rhodaniens

California Studies in Classical Antiquity =

Capitolium

Carinthia I. Geschichtliche und

volkskundliche Beiträge zur

Heimatkunde Kärntens

Carnuntum-Jahrbuch. Zeitschrift für Archäologie und Kulturgeschichte des Donauraumes

Carpica. Muzeul judeţean de istorie şi = artă, Bacău

Il Carrobbio. Rivista di studi bolognesi =

CEDAC. Bulletin. Centre d'études et de = documentation archéologique de la

Conservation de Carthage

Celticum. Supplément à Ogam

Cercetări numismatice. Muzeul naţional = de istorie

Chiron. Mitteilungen der Kommision für $=$ Alte Geschichte und Epigraphik des

Deutschen Archäologischen Instituts

Chronique d'Egypte

Ciceroniana

The Cincinnati Art Museum Bulletin

Civiltà classica e cristiana

Civiltà padana. Archeologia e storia del territorio

Classica et mediaevalia. Revue danoise de $=$ philologie et d'histoire

Classical Antiquity

The Classical Journal

Classical Philology

The Classical Quarterly

The Classical Review

Clio. Revista do Centro de história da Universidade de Lisboa

Coin Hoards. The Royal Numismatic Society, London

Colloqui del sodalizio

Communicationes archaeologicae hungaricae

Communicationes. Rei cretariae romanae $=$ fautores

Comptes rendus des séances de l'Académie des inscriptions et belles-
$=$ CahMusChampollion
$=\quad$ CahLig

CArch

CahASubaqu

CahByrsa

CahCerEg

CahDelFrIran

CahKarnak

CahMariemont

CahPEg

CahTun

CahRhodBord

CalifStClAnt

Capitolium

Carinthia I

Carnuntumjb

Carpica

Carrobbio

CEDAC

Celticum

CercNum

Chiron

ChronEg

Ciceroniana

CincArtB

$\mathrm{CivClCr}$

CivPad

ClMediaev

ClAnt

$\mathrm{ClJ}$

CIPhil

$\mathrm{ClQ}$

ClR

Clio

Coin Hoards

ColloquiSod

CommunicAHung

CommunicReiCret

CRAI
CahArmeeRom 


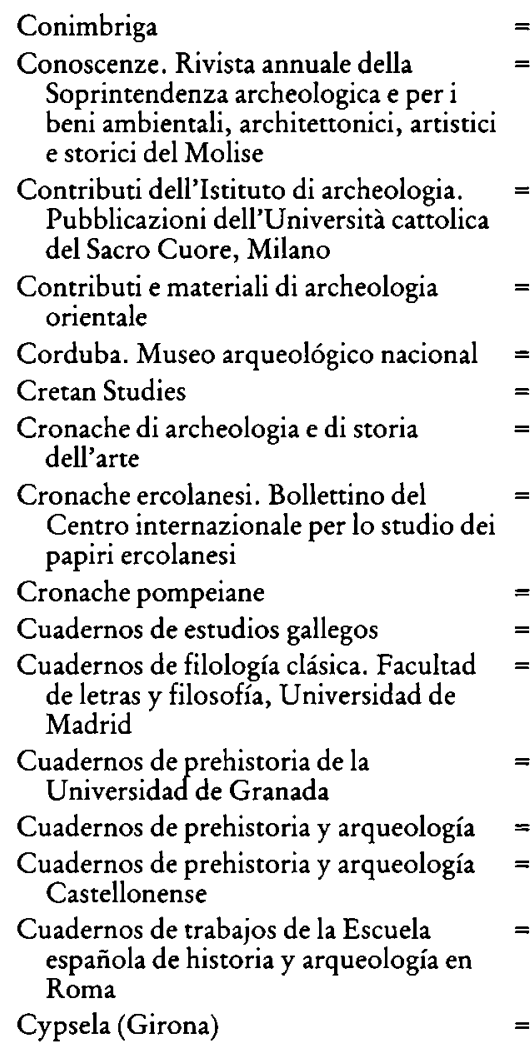

Dacia

Dacoromania. Jahrbuch für östliche Latinität

Damaszener Mitteilungen

A Debreceni Déri múzeum évkönyve

Dédalo. Revista de arte e arqueologia

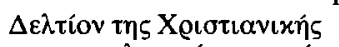

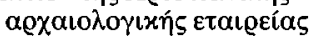

Denkmalpflege in Baden-Württemberg

Deutsche Kunst und Denkmalpflege

Deutsche Literaturzeitung für Kritik der internationalen Wissenschaft

Diadora

Dialoghi di archeologia

Dialogues d'histoire ancienne

Dioniso

Documenta albana

Documenti. Istituto per i beni artistici, culturali, naturali della regione EmiliaRomagna

Documents d'archéologie méridionale =

Documents d'archéologie régionale. = Université catholique de Louvain

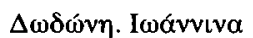

Les dossiers de l'archéologie

Les dossiers du Centre régional archéologique d'Alet

Dumbarton Oaks Papers

Durius. Boletín castellano de estudios clásicos

$=$
$=$
$=$
$=$
$=$
$=$
$=$
$=$
$=$
$=$
$=$
$=$
$=$
$=$
$=$
$=$
$=$
$=$

Conimbriga

CIstAMilano

CMatAOr

Corduba

CretSt

CronAStorArt

CronErcol

CronPomp

CuadGallegos

CuadFilCl

CuadGranada

CuadPrehistA

CuadCastellon

CuadRom

Cypsela

DebrecMuzEvk

Dédalo

DeltChrA

DenkmPflBadWürt

DKuDenkmPfl

DLZ

Diadora

DialA

DialHistAnc

Dioniso

DocAlb

DocEmRomag

DocAMerid

DocALouv

Dodone

DossAParis

DossAlet

DOP

Durius
East and West

Eburodunum

Echos du monde classique. Classical views

Egitto e Vicino Oriente

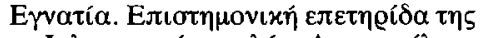

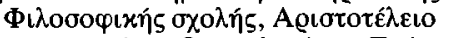

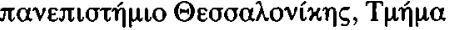

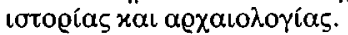

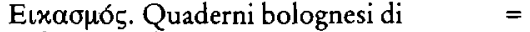
filologia classica

Eirene. Studia graeca et latina antico

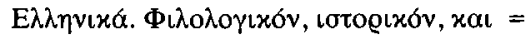

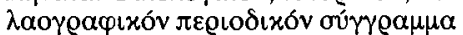

Emerita

Emilia preromana

Empúries

Enchoria. Zeitschrift für Demotistik und = Koptologie

Eos. Commentarii Societatis philologae = Polonorum

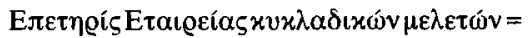

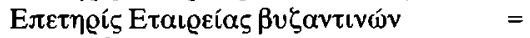
oлovó́v

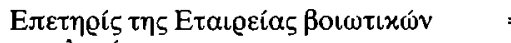

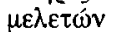

Epigraphica
Epigraphica Anatolica. Zeitschrift für $=$ Epigraphik und historische Geographie Anatoliens

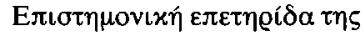

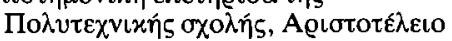

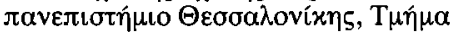

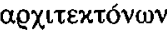

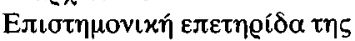

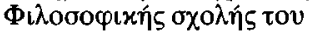

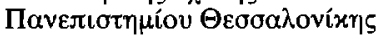

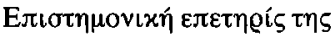

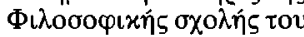

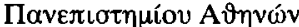

Eranos. Acta philologica suecana

Eranos-Jahrbuch

Eretz-Israel

Estudios de arqueología alavesa

Estudios de prehistoria y arqueología madrileñas

Estudios del Seminario de prehistoria, arqueología e historia antigua de la Facultad de filosofía y letras de Zaragoza

Ethnos. Revista do Instituto português de = arqueologia, história e etnografia

Etruscans. Bulletin of the Etruscan Foundation

Les études classique

Etudes classiques. Faculté des lettres et sciences humaines d'Aix

Etudes d'archéologie classique =

Etudes et travaux. Studia i prace. Travaux = du Centre d'archéologie méditerranéenne de l'Académie des sciences polonaise

Eunomia. Ephemeridis Listy filologické = supplementum

Euphrosyne. Revista de filologia clássica =
Etudes de papyrologie =

Etudes sur Pézenas et l'Hérault =
EastWest Eburodunum

Echos Cl

EgVicOr

Egnatia

Eikasmos

Eirene

Elenchos

Ellenika

Emerita

EmPrerom

Empúries

Enchoria

Eos

EpetKyklMel

EpetByzSpud

EpetBoiotMel

Epigraphica

EpigrAnat

$=$ EpistEpetPolytThess

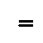

EpistEpetThess

EpistEpetAth

Eranos

Eranos Jb

ErIsr

EstAAlava

EstMadr

EstZaragoza

Ethnos

Etruscans

$\mathrm{EtCl}$

EtClAix

EtACl

EtP

Et'Trav

EtPézenas

Eunomia

Euphrosyne 
Expedition

Extremadura arqueológica

Faenza

Faventia. Facultat de lletres, Barcelona

Felix Ravenna

Das Fenster in der Halle der

Kreissparkasse Köln

Ficheiro epigráfico. Suplemento de "Conimbriga“"

Figlina. Documents du Laboratoire de céramologie de Lyon

Fogg Art Museum. Acquisitions 19xx

Folia archaeologica

Folia orientalia

Fonaments. Prehistòria i mon antic als Paisos Catalans

Fondamenti. Rivista quadrimestrale di cultura

Fontes archaeologici Posnanienses

Fornvännen. Tidskrift för svensk antikvarisk forskning

Forschungen und Berichte. Staatliche Museen zu Berlin

Forschungen und Berichte zur Vor- und Frühgeschichte in Baden-Württemberg

Forschungen und Fortschritte

Forum. Revue du Groupe d'archéologie antique

Frühmittelalterliche Studien. Jahrbuch = des Instituts für Frühmittelalterforschung der Universität Münster

Fundberichte aus Baden-Württemberg =

Fundberichte aus Hessen

Fundberichte aus Österreich

Fundberichte aus Schwaben

Gaceta numismática (Barcelona)

Gades. Revista del Colegio universitario de filosofía y letras

Gallaecia (Santiago de Campostela)

Gallia. Fouilles et monuments

archéologiques en France

métropolitaine.

Gallia informations. Préhistoire et histoire

Gallia préhistoire

Gazette des beaux-arts

Genava

Geo-archeologia. Periodico dell'Associazione geo-archeologica italiana

Gerión (Madrid)

Germania

Gesta. International Center of Medieval Art

Giglio di roccia

Giornale filologico ferrarese

Giornale italiano di filologia

Expedition

ExtremA

$=$

$=$ $=$

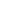

$=$
FuBerBadWürt FuBerHessen FuBerÖ
FuBerSchwab

Faenza

Faventia

FelRav

Das Fenster

FichEpigr

Figlina

FoggArtMusAcqu

FolA

FolOr

Fonaments

Fondamenti

FontAPos

Fornvännen

FuB

FBerBadWürt

FuF

Forum

FrühMitAltSt
GacNum

Gades

Gallaecia

Gallia

GalliaInf

GalliaPrehist

GazBA

Genava

Geo-archeologia

Gerión

Germania

Gesta

GiRoccPalermo

GiornFilFerr

GiornItFil
Giornale storico della Lunigiana

Glasgow Archaeological Journal

Glasnik Zemaljskog muzeja u Sarajevu

Glotta

Gnomon

Godišen zbornik na Filozofskiot fakultet = na Universitetot vo Skopje

Godišnik na Narodnija arheologičeski muzej Plovdiv. Annuaire du Musée national archéologique Plovdiv

Goriški letnik. Zbornik Goriškega muzeja

Göttinger Miszellen. Beiträge zur ägyptologischen Diskussion

Göttingische Gelehrte Anzeigen Pula

Graecolatina et orientalia. Zborník Filozofickej fakulty Univerzity Komenského

Graecolatina Pragensia

Grazer Beiträge

Greece and Rome

Greek, Roman and Byzantine Studies

Gymnasium

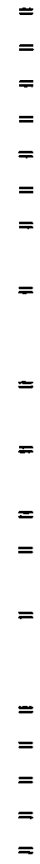

Glasnik. Srpsko arheološko društvo

Građa i rasprave. Arheološki muzej Istre, =

Habis. Universidad de Sevilla. Arqueología, filología clásica

Hamburger Beiträge zur Archäologie

Hamburger Beiträge zur Numismatik

Harvard Studies in Classical Philology

The Harvard Theological Review

Hefte des Archäologischen Seminars der Universität Bern

Helike. Universidad nacional de educación a distancia, Centro regional de Elche

Helikon

Helinium

Helios. A journal devoted to critical and = methodological studies of classical culture, literature, and society

Hellenika. Jahrbuch für die Freunde Griechenlands

Helvetia archaeologica

Hephaistos. Kritische Zeitschrift zur Theorie und Praxis der Archäologie und angrenzender Wissenschaften

Hermes. Zeitschrift für klassische Philologie

Hesperia. Journal of the American School = of Classical Studies at Athens

Hispania antiqua epigraphica

Hispania antiqua. Revista de historia antigua

Hispania epigraphica

Hispania. Revista española de historia

Historia. Zeitschrift für Alte Geschichte =

Historica. Academia RSR, Centrul de = istorie, filologie şi etnografie din Craiova

Historische Sprachforschung
GiornStorLun

Glas AJ

GlasBeograd

GlasSarajevo

Glotta

Gnomon

GodZborSkopje

GodMuzPlov

GorLet

GöttMisz

GGA

GraRaspr

GrLatOr

GrLatPrag

GrazBeitr

$\mathrm{GaR}$

GrRomByzSt

Gymnasium

Habis

HambBeitrA

HambBeitrNum

HarvStClPhil

HarvTheolR

HefteABern

Helike

Helikon

Helinium

Helios

HellenikaJb

HelvA

Hephaistos

Hermes

Hesperia

HispAntEpigr

HispAnt

HispEpigr

Hispania

Historia

Historica

HistSprF 
Historische Zeitschrift

Histria archeologica

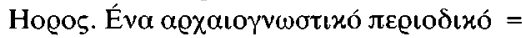

Huelva arqueológica

Humanistische Bildung

Humanitas

Ilerda. Instituto de estudios ilerdenses = (Lérida)

Iliria

Illinois Classical Studies

The Illustrated London News

Index. Quaderni camerti di studi romanistici

Indiana University Art Museum Bulletin =

Indice histórico español

Indogermanische Forschungen

Insula Fulcheria

International Journal of Nautical Archaeology

Invigilata lucernis

Iran. Journal of the British Institute of Persian Studies

Iranica antiqua

Iraq

Israel Exploration Journal

The Israel Museum Journal

The Israel Museum News

Israel Numismatic Journal

Istanbul Arkeoloji müzeleri yıllığı

Istanbuler Mitteilungen

Italia nostra

Italica. Cuadernos de trabajos de la

Escuela española de historia y arqueología en Roma -

Izvestija na Arheologičeskija institut. Bulletin de l'Institut archéologique bulgare

Izvestija na muzeite ot Južna Bălgarija. Bulletin des musées de la Bulgarie du Sud

Izvestija na Narodnija muzej Burgas

Izvestija na Narodnija muzej Varna

The J. Paul Getty Museum Journal

Jaarbericht van het Vooraziatischegyptisch genootschap Ex Oriente Lux

Jábega. Revista de la Disputación provincial de Málaga

Jadranski zbornik. Prilozi za povijest Istre, Rijeke i Hrvatskog primorja

Jahrbuch der Akademie der Wissenschaften in Göttingen

Jahrbuch. Bayerische Akademie der Wissenschaften

Jahrbuch der Berliner Museen

Jahrbuch des Bernischen Historischen

Museums in Bern

Jahrbuch des Deutschen Archäologischen Instituts
HistriaA

Horos

HuelvaA

HumBild

Humanitas

IndUnArtB IndiceHistEsp Indogerm $F$ InsFulc IntJNautA

InvLuc Iran

IranAnt IsrExplJ IsrMusJ IsrMusN IsrNumJ

IstanbAMüzYil IstMitt ItNostr Italica

BIBulg

Jábega

Jadr'Zbor

JbGött

JbMünch

JbBerlMus

JbBernHistMus

JdI
Jahrbuch des Museums für Kunst und Gewerbe in Hamburg

Jahrbuch des Oberösterreichischen Musealvereins

Jahrbuch des Römisch-germanischen Zentralmuseums Mainz

Jahrbuch für Antike und Christentum

Jahrbuch für kleinasiatische Forschung

Jahrbuch für Numismatik und Geldgeschichte

Jahrbuch für prähistorische und ethnographische Kunst

Jahrbuch der Hamburger Kunstsammlungen

Jahrbuch der Kunsthistorischen Sammlungen in Wien

Jahrbuch der Österreichischen Byzantinistik

Jahrbuch Preussischer Kulturbesitz

Jahrbuch der Schweizerischen Gesellschaft für Ur- und Frühgeschichte

Jahrbuch der Staatlichen Kunstsammlungen in BadenWürttemberg

Jahresbericht der Archäologischen Bodenforschung des Kantons BaselStadt

Jahresbericht der Bayerischen Bodendenkmalpflege

Jahresbericht des Instituts für Vorgeschichte der Universität Frankfurt a. M.

Jahresbericht. Gesellschaft Pro Vindonissa

Jahresbericht. Schweizerisches Landesmuseum Zürich

Jahresberichte aus Augst und Kaiseraugst

Jahreshefte des Österreichischen Archäologischen Institutes in Wien

Jahresschrift für mitteldeutsche Vorgeschichte

Journal des savants

Journal of the American Research Center = in Egypt

Journal of Archaeological Science

Journal of Cuneiform Studies

The Journal of Egyptian Archaeology

Journal of Field Archaeology

Journal of Glass Studies

The Journal of Hellenic Studies

Journal of Juristic Papyrology

Journal of Mediterranean Anthropology and Archaeology

Journal of Mediterranean Archaeology

Journal of Mithraic Studies
Journal of Near Eastern Studies

Journal of Prehistoric Religion

Journal of Roman Archaeology

Journal of Roman Military Equipment Studies

Journal of Roman Pottery Studies

The Journal of Roman Studies

Journal of Semitic Studies

The Journal of the Walters Art Gallery
$=$

$=$

$=$

$=$

$=$

$=$

$=$

$=$

$=$

$=$

$=$

$=$

$=$

$=$

$=$

$=$

JberBayDenkmPfl

JberVgFrankf

JberProVindon

JberZürich

JberAugst

ÖJh

JSchrVgHalle

JSav

JARCE

JASc

JCunSt

JEA

JFieldA

JGS

JHS

JjurP

JMedAnthrA

JMedA

JMithrSt

JNES

JPrehistRel

JRA

JRomMilSt

JRomPotSt

JRS

JSemSt

JWaltersArtGal 
Journal of the Warburg and Courtaud = Institutes

Jura. Rivista internazionale di diritto= romano e antico.

Kadmos

Kairos. Zeitschrift für Judaistik und = Religionswissenschaft

Karthago. Revue d'archéologie africaine =

Kêmi. Revue de philologie et d'archéologie égytiennes et coptes

Kent Archaeological Review

Kernos. Revue internationale et pluridisciplinaire de religion grecque antique

Klearchos

Klio

Kobie. Grupo espeleológico vizcaíno (Bilbao)

Koinonia

K $\Omega K A \wedge O \Sigma$. Studi pubblicati

dall'Istituto di storia antica dell'Università di Palermo

Kölner Jahrbuch für Vor- und Frühgeschichte

Kratkie soobščenija o dokladach i polevych issledovanijach Instituta archeologii

Kratkie soobščenija Instituta archeologii

Kratylos. Kritisches Berichts- und Rezensionsorgan für indogermanische und allgemeine Sprachwissenschaft

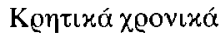

Ktema. Civilisations de l'Orient, de la =

Grèce et de Rome antique

Kuml

Kungl. vitterhets historie och antikvitets akademiens handlingar. Antikvariska serien

Kunst des Orients

Kunst der Welt in den Berliner Museen

Kunstchronik

Kunstgeschichtliche Anzeigen

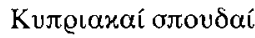

Kush. Journal of the Sudan Antiquities Service

Labeo. Rassegna di diritto romano

Lampas. Tijdschrift voor nederlandse classici

Landeskundliche Vierteljahrsblätter. Trier =

Langues orientales anciennes. Philologie = et linguistique.

Latinitas

Latomus. Revue d'études latines

Levant. Journal of the British School of Archaeology in Jerusalem and the British Institute at Amman for Archaeology and History

Libya antiqua

XXXIV
$=$

$=$

$=$

$=$

$=$

$=$

$=$

$=$

$=$

$=$

$=$

KölnJbVFrühGesch

$=$

$=$

$=$

Labeo

Lampas

LandKunVierJBl

LangOrAnc

Latinitas

Latomus

Levant

LibyaAnt

KentAR

Kernos

learchos

Klio

Koinonia

Kokalos

KraSoob

KraSoobInstA

Kratylos

KretChron

Kuml

Handlingar

$\mathrm{KuOr}$

Kunstchronik

KuGeschAnz

ypSpud

Kush
Libyan Studies

Libyca. Bulletin du Service des antiquités. = Archéologie, épigraphie

Libyca. Travaux du Laboratoire d'anthropologie et d'archéologie préhistorique du Musée du Bardo

Linguistica, epigrafia, filologia italica

Listy filologické

Lucentum. Anales de la Universidad de Alicante. Prehistoria, arqueología e historia antigua

Lustrum

Macedoniae acta archaeologica

Madrider Mitteilungen

Maia

Mainake

Mainzer Zeitschrift

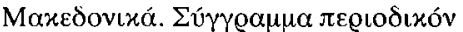

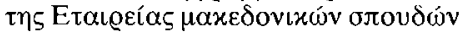

Marburger Winckelmann-Programm

Mari. Annales de recherches interdisciplinaires

Marisia. Studii şi materiale. Arheologie, istorie, etnografie

Marsyas. Studies in the History of Art =

Masca Journal. Museum Applied Science = Center for Archaeology, University of Pennsylvania

Masca Research Papers in Science and Archaeology

Materiale şi cercetări arheologice

Materiali e discussioni per l'analisi dei testi classici

Materialy i issledovanija po archeologii SSSR

Materialy po archeologii BSSR

Materialy po archeologii severnogo Pričernomor'ja

Materiały starożytne

Materiały wczesnośredniowieczne

Meander. Miesięcznik poświęcony

kulturze świata starożytnego

Meddelanden fran Lunds Universtitets Historiska Museum

Meddelelser fra Ny Carlsberg Glyptotek Kabenhavn

Meddellelser fra Thorwaldsens Museum =

Mededelingen van het Nederlands = Instituut te Rome. Antiquity

Medelhavsmuseet. Bulletin (Stockholm) =

Medieval Archaeology. Journal of the = Society for Medieval Archaeology

Mediterranean Archaeology

Mediterranean Historical Review

Mélanges de la Casa de Velázquez. Antiquité et moyen âge

Mélanges de l'Ecole française de Rome. Antiquité

Mélanges de l'Université Saint-Joseph
LibSt

LibycaBServAnt

Libyca'Trav

LingIt

LF

Lucentum

Lustrum

MacActaA

$\mathrm{MM}$

Maia

Mainake

MainzZ

Makedonika

MarbWPr

Mari

Marisia

Marsyas

MascaJ

MascaP

MatCercA

Mat'TestiCl

MatIsslA

MatABelorSSR

MatASevPrič

MatStar

MatWczes

Meander

MeddelLund

MeddelGlypt

MeddelThor

MededRom

MedelhavsMusB

MedievA

MedA

MedHistR

MelCasaVelazquez

MEFRA

MelBeyrouth 
Mémoires de l'Académie des inscriptions = et belles-lettres

Mémoires de l'Institut national de France =

Mémoires de la Société nationale des= antiquitaires de France

Memoirs of the American Academy in = Rome

Memoria antiquitatis. Acta Musei

Petrodavensis. Revista Muzeului arheologic Piatra Neam

Memoria. Universidad de Barcelona, Instituto de arqueología y prehistoria.

Memorias de historia antigua. Universidad de Oviedo

Memorias de los museos arqueologícos=

Memorie. Atti della Accademia nazionale = dei Lincei, Classe di scienze morali, storiche e filologiche

Memorie. Atti della Ponteficia accademia = romana di archeologia

Memorie dell'Accademia di archeologia, = lettere e belle arti di Napoli

Memorie storiche forogiuliesi

Mesopotamia. Rivista di archeologia

Messana. Rassegna di studi filologici, = linguistici e storici

Métis. Revue d'anthropologie du monde = grec ancien

Metropolitan Museum Journal

The Metropolitan Museum of Art = Bulletin

Minia (Braga)

Minos

Miscellanea di studi di letteratura cristiana = antica

Miscellánies arqueològiques sobre Mataró = i el Maresme

Mitteilungen der Anthropologischen = Gesellschaft in Wien

Mitteilungen der Archäologischen Gesellschaft Graz

Mitteilungen des Archäologischen Instituts der Ungarischen Akademie der Wissenschaften

Mitteilungen des Deutschen Archäologen-Verbandes e. V.

Mitteilungen des Deutschen

Archäologischen Instituts, Abteilung Kairo

Mitteilungen des Deutschen Archäologischen Instituts, Athenische Abteilung

Mitteilungen des Deutschen

Archäologischen Instituts, Römische Abteilung

Mitteilungen des Instituts für Orientforschung

Mitteilungen des Kunsthistorischen Instituts Florenz

Mitteilungen der Deutschen OrientGesellschaft zu Berlin

Mitteilungen. Leichtweiss-Institut für Wasserbau der Technischen Universität Braunschweig.

Mitteilungen der Österreichischen = Numismatischen Gesellschaft
MemAcInscr

MemInstNatFr

MemAntFr

MemAmAc

MemAnt

MemBarcelA

MemHistAnt

MemMusAProvinc

MemLinc

MemPontAc

MemNap

MemStorFriuli

Mesopotamia

Messana

Métis

MetrMus]

BMetrMus

Minia

Minos

MiscCrAnt

MiscAMataro

MAnthrWien

MAGesGraz

MAInstUngAk

MDAVerb

MDIK

AM

$\mathrm{RM}$

MIO

MKuHistFlorenz

MDOG

MInstWasser

MÖNumGes
Mitteilungen der Prähistorischen Kommission der Österreichischen

Akademie der Wissenschaften

Mitteilungen zur Kulturkunde

Mitteilungsblatt der Schweizerischen Gesellschaft für Ur- und Frühgeschichte

Mitteilungsblatt für Vor- und Frühgeschichte

Mnemosyne

Monumentet

Monumenti antichi

Monuments et mémoires. Fondation $E$. Piot

A Móra Ferenc múzeum évkönyve

Münchener Studien zur Sprachwissenschaft

Münchner Jahrbuch der bildenden Kunst =

Münstersche Beiträge zur antiken Handelsgeschichte

Murcia. Boletin informativo de la Excma. = Diputación provincial

Muse. Annual of the Museum of Art and = Archaeology, University of Missouri, Columbia

Museen in Köln. Bulletin

Musei e gallerie d'Italia

Museo civico Pontecorvo. Quaderni

El Museo de Pontevedra

Museo de Zaragoza. Boletín

Le muséon. Revue d'études orientales

Museum Africum. West African Journal of Classical and Related Studies

Museum criticum

Museum Haaretz, Tel Aviv. Yearbook

Museum helveticum

Museum Notes. The American

Numismatic Society

Museum. A quarterly review published by UNESCO

Museum Tusculanum. København

Muzei i pametnici na kulturata

Muzeul naţional. Bucareşti

Nachrichten der Akademie der

Wissenschaften in Göttingen

Napoli nobilissima

Nassauische Annalen

Nestor

Neue Ephemeris für semitische Epigraphik

News Bulletin and Calendar. Worcester Art Museum

Newsletter. Department of pottery technology, University of Leiden

Nikephoros. Zeitschrift für Sport und Kultur im Altertum

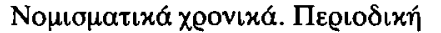

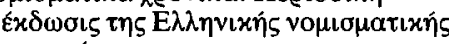

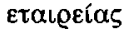

Norba. Revista de arte, geografía e historia (Cáceres)

Noticiario arqueológico hispánico. Arqueología

Noticiario arqueológico hispánico. Prehistoria

Notiziario. Museo civico, Associazione = archeologica, Allumiere
MPrähistKomWien

$=$

MSchwUrFruhr

MKul

$=$

MBIVFrühGesch

Mnemosyne

Monumentet

MonAnt

MonPiot

MuzEvkSzeged

MünchStSprWiss

MüJb

MünstBeitr

Murcia

Muse

MusKöln

MusGalIt

QuadMusPontecorvo

MusPontevedra

BMusZaragoza

Muséon

MusAfr

MusCrit

MusHaaretz

MusHelv

MusNotAmNumSoc

MuseumUnesco

MusTusc

MuzPamKul

MuzNaţ

$=$
$=$

$\begin{array}{rr}= & \text { NachrAkGött } \\ = & \text { NapNobil } \\ = & \text { NassAnn } \\ = & \text { Nestor } \\ = & \text { NEphemSemEpigr }\end{array}$

NBWorcArtMus

NewsletterPotTech

Nikephoros

NomChron

Norba

NotAHisp

NotAHispPrehistoria

NotAllumiere 
Notiziario. Soprintendenza archeologica $=$ della Lombardia

Notizie degli scavi di antichità

Notizie del Chiostro del Monastero maggiore. Rassegna di studi del Civico museo archeologico e del Civico gabinetto numismatico di Milano

La nouvelle Clio

Novaensia. Badania Ekspedycji archeolgicznej Uniwersytetu warszawskiego w Novae

Nubia christiana

Nubian Letters

Numantia. Investigaciones arqueológicas $=$ en Castilla y León

Numen

Numisma. Revista de la Sociedad iberoamericana de estudios numismáticos

The Numismatic Chronicle

Numismatica

Numismatica e antichità classiche. Quaderni ticinesi

Numismatische Zeitschrift

Numizmatičar

Numizmatika i epigrafika

Nummus. Sociedade portuguesa de numismatica

Nuovi annali della Facoltà di magistero dell'Università di Messina

Nuovo Bullettino archeologico sardo

Nuovo Didaskaleion
Österreichische Akademie der Wissenschaften, Philosophischhistorische Klasse. Denkschriften

Oudheidkundige mededelingen uit het

Oxford Journal of Archaeology

NouvClio

Novaensia

Oxford Studies in Ancient Philosophy Rijksmuseum van oudheden te Leiden

DenkschrWien

OudhMeded

OxfJA

OxfStPhilos

Pact

Nublet

Numantia

Numen

Numisma

NumChron

NumismaticaRom

NumAntCl

NumZ

Numizmatičar

NumEpigr
Pact. Revue du Groupe européen d'études = pour les techniques physiques, chimiques et mathématiques appliquées à l'archéologie

Padusa. Bollettino del Centro polesano di = studi storici, archeologici ed etnografici, Rovigo

Paideuma. Mitteilungen zur Kulturkunde =

Palaeohistoria

Palestine Exploration Quarterly =

Palladio. Rivista di storia dell'architettura =

Pallas. Annales publiées par la Faculté des = lettres et sciences humaines de Toulouse

Památky archeologické

Pan. Studi dell'Istituto di filologia latina =

Pantheon

La parola del passato

Partenope

Peristil. Zbornik radova za povijest = umjetnosti

Persica

Peuce. Studii şi comunicări de istorie şi arheologie

Philologus. Zeitschrift für das klassische = Altertum

Phoenix. The Classical Association of Canada

Phoenix. Bulletin uitgegeven door het = Vooraziatisch-Egyptisch Genootschap Ex Oriente Lux

Phoibos

Phronesis

Picus. Studi e ricerche sulle Marche nell'antichità

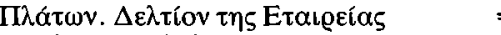
$\varepsilon \lambda \lambda \eta \dot{v} \omega \nu v \varphi \lambda{ }^{\prime} \lambda \sigma \gamma \omega v$

Poetica

Pompeii, Herculaneum, Stabiae. Amici di Pompei

Pontica

Portugalia

Prace archeologiczne

Prace i materialy Muzeum archeologicznegoietnograficznegowłodzi

Praehistorische Zeitschrift

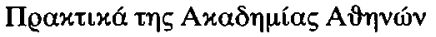

$=$

$=$

$=$

$=$

$=$
$=$

$=$

$=$

$=$ $=$
Papers of the British School at Rome

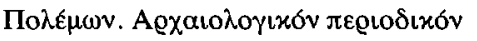

Nummus

AnnMessMag

BASard

NuovDidask

OccasPublClst

Offa

Ogam

Oikumene

Opus

OpAth

OpuscFin

OpRom

Oretania

OA

OrChr

Orient

OrChrPer

Orientalia

OLZ

Origini

OrpheusThracSt

Orpheus

OsjZbor

OstbGrenzm Bollettino. Associazione internazionale Volkskunde 


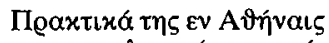

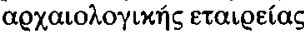

Préhistoire

Preistoria alpina. Museo tridentino di scienze naturali

Prilozi. Centar za povijesne znanosti Sveučilišta u Zagrebu, Odjel za arheologiju

Príncipe di Viana

Pro Austria romana

The Proceedings of the African Classical Associations

Proceedings of the Cambridge Philological Society

Proceedings of the Prehistoric Society

Prometheus. Rivista quadrimestrale di studi classici

Prospettiva. Rivista di storia dell'arte antica e moderna

Prospezioni archeologiche. Quaderni

Prospezioni. Bollettino di informazioni scientifiche

Provence historique

La Provincia di Lucca

Pulpudeva. Semaines philippopolitaines de l'histoire et de la culture thrace

Puteoli. Studi di storia antica

Pyrenae. Crónica arqueológica

Quaderni catanesi di studi classici e medievali

Quaderni. Centro studi lunensi

Quaderni d'archeologia reggiana

Quaderni del Dipartimento delle arti, Università della Calabria

Quaderni della Soprintendenza archeologica del Piemonte

Quaderni dell'Istituto di archeologia della = Facoltà di lettere e filosofia della Università di Messina.

Quaderni dell'Istituto di archeologia e storia antica, Università di Chieti

Quaderni dell'Istituto di filologia greca

Quaderni dell'Istituto di lingua e letteratura latina, Università di Roma

Quaderni di archeologia del Veneto

Quaderni di archeologia della Libia

Quaderni di protostoria

Quaderni di storia

Quaderni erbesi. Civico museo archeologico di Erba

Quaderni. Istituto di lingue e letterature classiche, Facoltà di magistero, Universtà degli studi, Lecce

Quaderni. Soprintendenza archeologica per le provincie di Cagliari e Oristano

Quaderni urbinati di cultura classica

Quaderns d'arqueologia i història de la ciutat (Barcelona)

Quaderns d'estudis medievals

Quaternaria

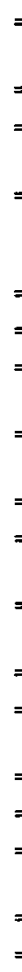

$=$

ProcCambrPhilSoc

ProcPrehistSoc

Prometheus

Prospettiva

ProspAQuad

Prospezioni

ProvHist

ProvLucca

Pulpudeva

Puteoli

Pyrenae

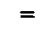$$
=
$$

QuadStLun QuadAReggio QuadACal

QuadAPiem

QuadMess

QuadChieti

QuadIstFilGr

QuadIstLat

QuadAVen

QuadALibia

QuadProtost

QuadStor

QuadErb

QuadLecce

QuadACagl

QuadUrbin

QuadABarcel

QuadEstMediev

Quaternaria
Radiocarbon
Radovi Instituta Jugoslavenske akademije $=$ znanosti i umjetnosti u Zadru

Raggi. Zeitschrift für Kunstgeschichte = und Archäologie

Rassegna del Lazio

Rassegna di archeologia. Associazione = archeologica piombinese

Rassegna storica salernitana

Ratiariensia. Studi e materiali mesici e danubiani

Raydan. Journal of the ancient Yemeni antiquities and epigraphy

Recherches albanologiques

Recherches archéologiques. Institut
d'archéologie de l'Université de d'archéol
Cracovie

Recueil des notices et mémoires de la Société archéologique du département de Constantine

Reden und Gedenkworte. Orden Pour le = mérite für Wissenschaften und Künste

Rei cretariae romanae fautorum acta

P $\eta \mu \alpha$

Rendiconti. Atti della Pontificia accademia romana di archeologia

Rendiconti della Accademia di archeologia, lettere e belle arti, Napoli

Rendiconti. Istituto lombardo, Accademia di scienze e lettere

Report of the Department of Antiquities, = Cyprus

Report on the Working of the Museum Department for the Year 19xx. Malta, Department of information

Reppal. Centre d'études phéniciennespuniques et des antiquités libyques, Tunis

Revista da Facultade de letras. Serie de = historia. Universidade do Porto

Revista de archivos, bibliotecas $\mathrm{y}$ museos =

Revista de Girona

Revista de Guimarães

Revista. Instituto de estudios alicantinos

Revue archéologique

Revue archéologiques de l'Est et du= Centre-Est consacrée aux antiquités nationales

Revue archéologique de Narbonnaise

Revue archéologique du Centre de la France

Revue belge de numismatique et de sigillographie

Revue belge de philologie et d'histoire

Revue biblique

Revue d'archéométrie

Revue d'assyriologie et d'archéologie orientale

Revue de l'histoire des religions

Revue de philologie, de littérature et d'histoire anciennes

Revue de la Saintonge et de l'Aunis

Revue d'égyptologie
Radiocarbon

RadAkZadar

Raggi

RassLazio

RassAPiomb

RassStorSalern

Ratiariensia

Raydan

RechAlb

RechACrac

RecConstantine

RedenGedenk

ReiCretActa

Rema

RendPontAc

RendNap

RendIstLomb

RDAC

RepMalta

Reppal

RPorto

RArchBiblMus

RGirona

RGuimar

RAlicante

RA

RAE

RANarb

RACFr

RBelgNum

RBelgPhilHist

$\mathrm{RBi}$

RArcheom

RAssyr

RHistRel

RPhil

RSaintonge

$\mathrm{REg}$ 
Revue des archéologues et historiens d'art = de Louvain

La revue des arts. Musées de France =

Revue des études anciennes

Revue des études arméniennes

Revue des études byzantines

Revue des études géorgiennes et caucasiennes

Revue des études grecques

Revue des études latines

La revue du Louvre et des musées de France

Revue hittite et asianique

Revue internationale des droits de l'antiquité

Revue numismatique

Das Rheinische Landesmuseum Bonn

Rheinisches Museum für Philologie

Ricerche e studi. Museo Francesco

Rivista archeologica dell'antica provincia e diocesi di Como

Rivista degli studi orientali

Rivista dell'Istituto nazionale d'archeologia e storia dell'arte

Rivista di archeologia

Rivista di archeologia cristiana

Rivista di cultura classica e medioevale

Rivista di filologia e d'istruzione classica

Rivista di scienze preistoriche

Rivista di studi bizantini e neoellenici

Rivista di studi classici

Rivista di studi fenici

Rivista di studi liguri

Rivista di studi marchigiani

Rivista di studi pompeiani

Rivista Ingauna e Intemelia

Rivista italiana di numismatica e scienze affini

Rivista storica calabrese

Rivista storica dell'antichità

Rocznik Muzeum narodowego w Warszawie

Romana gens. Bollettino dell'Associazione archeologica romana

Romanobarbarica. Contributi allo studio = dei rapporti culturali tra il mondo latino e mondo barbarico

Römische Quartalschrift für christliche = Altertumskunde und

Kirchengeschichte

Römisches Österreich

Rudiae. Ricerche sul mondo classico

Saalburg-Jahrbuch. Bericht des Saalburg- = Museums

Saeculum. Jahrbuch für Universalgeschichte

Saggi fenici

Saguntum. Papeles del Laboratorio de $=$ arqueología de Valencia Ribezzo, Brindisi
RAArtLouv

RArtMus

REA

REtArm

REByz

RGeorgCauc

REG

REL

RLouvre

RHA

RDroitsAnt

RNum

RheinMusBonn

RhM

RicStBrindisi

RAComo

RStOr

RIA

$\operatorname{RdA}$

$\mathrm{RACr}$

RCulClMedioev

RFil

RScPreist

RStBiz

$\mathrm{RStCl}$

RStFen

RStLig

RStMarch

RStPomp

RIngIntem

RItNum

RStorCal

RStorAnt

RoczMuzWarsz

RomGens

RömQSchr

RömÖ

Rudiae

SaalbJb

Saeculum

SaggiFen

Saguntum
Romanobarbarica
Saitabi. Noticiario de historia, arte y

Saitabi

arqueología de Levante

Sandalion. Quaderni di cultura classica, cristiana e medievale

Sargetia. Acta Musei (regionalis) devensis = Savaria. Bulletin der Museen des Komitats = Vas

Sbornik prací Filosofické fakulty

Brněnské university. Rada archeologicko-klasická (E)

Schild von Steier. Beiträge zur Steirischen $=$ Vor- und Frühgeschichte und

Münzkunde

Schweizer Münzblätter

Schweizerische numimatische Rundschau $=$

Scienze dell'antichità. Storia, archeologia, = antropologia

Scripta classica Israelica. Yearbook of the = Israel Society for the Promotion of Classical Studies

Scripta Hierosolymitana. Publications of = the Hebrew University, Jerusalem

Scripta Mediterranea. Bulletin of the Society for Mediterranean Studies

Scrittura e civiltà

Setúbal arqueológica

Sibrium

Sicilia archeologica

Siculorum gymnasium

Sileno. Rivista di studi classici e cristiani $=$

Sintria. Revista de estudos de arqueologia, = arte e etnografia

Sitzungsberichte der Deutschen Akademie der Wissenschaften zu Berlin. Klasse für Sprache, Literatur und Kunst

Sitzungsberichte. Österreichische Akademie der Wissenschaften

Sitzungsberichte der Sächsischen Akademie der $W$ issenschaften zu Leipzig

Slovenská archeológia

Slovenská numizmatika

(Bulletin trimestriel de la) Société de géographie et d'archéologie (de la province) d'Oran

Soobščenija Gosudarstvennogo ordena Lenina Ërmitaža

Sovetskaja archeologija

Specimina nova dissertationum ex

Instituto historico Universitatis Quinqueecclesiensis de Iano Pannonio nominatae

Spoletium. Rivista di arte, storia, cultura. =

Städel-Jahrbuch

Stadion. Zeitschrift für Geschichte des Sports und der Körperkultur

Starinar. Arheološki institut, Beograd

Stiftung zur Förderung der

Erwerbungen

Strenna dei romanisti

Studi classici e orientali

Studi di antichità. Università di Lecce

Sandalion

Sargetia

Savaria

SborBrno

SchildStei

SchwMüBl

SchwNumRu

ScAnt

ScrClIsr

ScrHieros

ScrMed

ScrCiv

SetubalA

Sibrium

SicA

SicGymn

Sileno

Sintria

SBBerlin

SBWien

SBLeipzig

SlovA

SlovNum

SocGeoAOran

SoobErmit

SovA

SpNov

$=$

Spoletium

StädelJb

Stadion

Starinar

$=$ StiftHamburgKuSamml
Hamburgischen Kunstsammlungen.

XXXVIII 
Studi di egittologia e di antichità puniche =

Studi e documenti di archeologia

Studi eblaiti

Studi epigrafici e linguistici sul Vicino= Oriente antico

Studi etruschi

Studi genuensi

Studi italiani di filologia classica

Studi latini e italiani

Studi magrebini

Studi e materiali di storia delle religioni

Studi e materiali. Sopintendenza ai beni archeologici per la Toscana

Studi micenei ed egeo-anatolici

Studi romagnoli

Studi romani

Studi salentini

Studi sardi

Studi storico-religiosi

Studi tardoantichi

Studi trentini di scienze storiche. Sezione $=$ seconda

Studi urbinati, B. Scienze umane e sociali =

Studia aegyptiaca. Budapest

Studia archaeologica

Studia archeologiczne. Uniwersytet

Warszawski, Instytut archeologii

Studia et documenta historiae et iuris

Studia Oliveriana

Studia orientalia, Helsinki

Studia papyrologica

Studien zur altägyptischen Kultur

Studii clasice. Societatea de studii clasice din Republica socialistă Romănia

Studii şi cercetări de istorie veche şi arheologie

Studii şi cercetări de numismatică

Studium biblicum franciscanum. Liber annuus

Sumer

Sylva Mala. Bollettino del Centro di studi = archeologici di Boscoreale,

Boscotrecase e Trecase

Symbolae Osloenses

Syria. Revue d'art oriental et d'archéologie

Syro-Mesopotamian Studies

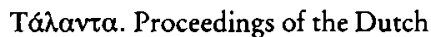
Archaeological and Historical Society

Taras. Rivista di archeologia

Teiresias. A review and continuing = bibliography of Boiotian studies

Tel Aviv. Journal of the Tel Aviv University Institute of Archaeology

Teruel. Instituto de estudios turolenses

Textiles anciens. Bulletin du Centre international d'étude des textiles anciens
StEgAntPun
StDocA
StEbla
StEpigrLing
StEtr
StGenu
StItFilCl
StLatIt
StMagreb
StMatStorRel
SteMat
SMEA
StRomagn
StRom
StSalent
StSard
StStorRel
StTardoant
StTrentStor

StUrbin

StAeg

StA

StAWarsz

StDocHistlur

StOliv

StOr

StP

StAltägKul

$\mathrm{StCl}$

StCercIstorV

StCercNum

StBiFranc

Sumer

Sylva Mala

SymbOslo

Syria

SyrMesopSt

Talanta

Taras

Teiresias

TelAvivjA

Teruel

TextilAnc
Theologische Rundschau

$\Theta \varepsilon \sigma \sigma \alpha \lambda$ เหर́

$\mathrm{H} \Theta \varepsilon \sigma \alpha \alpha \lambda o v i x \eta$

Thracia

Thraco-Dacica

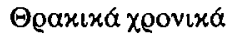

Tibiscus. Istorie, arheologie. Muzeul

Banatului Timişoara

Tierras de León

La Tour de l'Orle-d'Or, Semur-enAuxois

Trabajos de prehistoria

Trabalhos de antropologia e etnologia

Traditio. Studies in ancient and medieval history, thought and religion

Transactions and Proceedings of the American Philological Association

Transactions of the London and Middlesex Archaeological Society

Travaux de l'Institut d'art préhistorique, Université de Toulouse - Le Mirail

Travaux et mémoires. Centre de recherche $=$ d'histoire et civilisation byzantine.

Paris

Trésors monétaires

Trierer Winckelmannsprogramme

Trierer Zeitschrift für Geschichte und Kunst des Trierer Landes und seiner Nachbargebiete

Trudy Gosudarstvennogo Ėrmitaža =

Türk arkeoloji dergisi =

Tuy. Museo y archivo histórico diocesano =

Tyche. Beiträge zur Alten Geschichte, = Papyrologie und Epigraphik

Ugarit-Forschungen. Internationales

Jahrbuch für die Altertumskunde

Syrien-Palästinas

Uni-HH-Forschung.

Wissenschaftberichte aus der

Universität Hamburg

Ur-Schweiz. La Suisse primitive

L'Urbe. Rivista romana

Varstvo spomenikov istoričesko družestvo

Venezia arti. Bollettino del Dipartimento = di storia e critica delle arti dell'Università di Venezia.

Verbanus. Rassegna per la cultura, l'arte, = la storia del lago

Vereniging van vrienden Allard Pierson = museum Amsterdam.

Mededelingenblad

Vestnik drevnej istorii

Vetera Christianorum

Vichiana. Rassegna di studi filologici e = storici

Vicino Oriente

Vigiliae christianae

Visible Religion

Vizantijskij vremennik
TheolRu

Thessalika

Thessalonike

Thracia

Thraco-Dacica

ThrakChron

Tibiscus

TiLeon

TourOrleOr

TrabPrehist

TrabAntrEtn

Traditio

TransactAmPhilAss

TransactLond

TravToulouse

TravMem

TreMonet

TrWPr

$\operatorname{Tr} Z$

TrudyErmit

TürkAD

Tuy

Tyche

UgaritF

$=$ Uni-HH-Forschung

UrSchw

L'Urbe

VarSpom

Vekove

VenArt

Verbanus

VerAmstMeded

VesDrevIstor

VeteraChr

Vichiana

VicOr

VigChr

VisRel

VizVrem 
Vjesnik Arheološkog muzeja u Zagrebu =

Vjesnik za arheologiju i historiju dalmatinsku. Bulletin d'archéologie et d'histoire dalmates.

Wad-al-Hayara. Revista de estudios de la = Institución provincial de cultura

"Marqués de Santillana" de Guadalajara

The Walters Art Gallery Bulletin

Die Welt als Geschichte

Die Welt des Orients

Wiadomści archeologiczne. Bulletin archéologique polonais

Wiener Studien

Wiener Zeitschrift für die Kunde des Morgenlandes

Winckelmann-Gesellschaft, Stendal

Winckelmannsprogramm der Archäologischen Gesellschaft zu Berlin

Wissenschaftliche Mitteilungen des

Bosnisch-Herzegowinischen

Landesmuseums, A. Archäologie

Wissenschaftliche Zeitschrift der Friedrich-Schiller-Universität Jena

Wissenschaftliche Zeitschrift der Humboldt-Universität zu Berlin.

Gesellschafts- und

sprachwissenschaftliche Reihe

Wissenschaftliche Zeitschrift der Universität Rostock

Wissenschaftliche Zeitschrift. MartinLuther-Universität Halle-Wittenberg

World Archaeology

Würzburger Jahrbücher für die Altertumswissenschaft

Xenia. Semestrale di antichità

Yale Classical Studies

Yale University Art Gallery Bulletin
VjesAMuzZagreb

VjesDal

Wad-al-Hayara

\section{$=$}

$=$

$=$

$=$

$=$

$=$

$=$

$=$

$=$

(1)

$=$

$=$

$=$

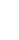

$=$
$=$

$=$

WürzbJb

BBaltimore

WeltGesch

WO

WiadA

WSt

WZKM

Jahresgabe

BWPr

WissMBosn

WissZJena

WissZBerl

WissZRostock

WissZHalle

WorldA

Xenia

YaleCISt

YaleUnivB

ZborZadar

Zbornik Instituta za historijske nauke u Zadru

Zbornik Narodnog muzeja Beograd

Zbornik radova Vizantološkog instituta. Recueil des travaux de l'Institut

d'études byzantines, Beograd

Zeitschrift des Deutschen Palästina-

Vereins

Zeitschrift der Deutschen

Morgenländischen Gesellschaft

$=$

$=$

Zeitschrift für ägyptische Sprache und Altertumskunde

Zeitschrift für die alttestamentliche Wissenschaft

Zeitschrift für Archäologie

ZborMuzBeograd ZborRadBeograd

ZDPV

$=$

ZDMG

ZÄS

ZAW

$\mathrm{XL}$ 